\title{
Utilização de FPGAs em Nuvem para o Ensino de Circuitos Integrados Reprogramáveis em um Curso de Engenharia de Computação
}

\author{
Vanessa R. O. da Silva ${ }^{1}$, Ricardo J. N. da Silveira ${ }^{1}$, Thomaz E. V. da Silva ${ }^{1,2}$, \\ Giovanni C. Barroso ${ }^{3}$ \\ ${ }^{1}$ Departamento de Teleinformática - Universidade Federal do Ceará (UFC) \\ Fortaleza - CE - Brasil \\ ${ }^{2}$ Centro de Educação - Instituto de Ensino e Pesquisa (INSPER) \\ São Paulo - SP - Brasil \\ ${ }^{3}$ Departamento de Física \\ Universidade Federal do Ceará (UFC) -Fortaleza - CE - Brasil \\ \{vanessarodrigues.vj, jardel.silveira, thomazveloso\}@gmail.com, \\ gcbefisica.ufc.br
}

\begin{abstract}
The use of FPGAs has been widespread in the market by enabling acceleration of workloads that require high processing power. However, the high cost of purchasing tools for using FPGAs compromises the teaching of this technology in universities. In this context, this paper presents a study on the use of cloud FPGAs in the teaching of reprogrammable integrated circuits in a Computer Engineering course. This study deals with the elaboration and application of laboratory practices. The results obtained took into account the feedback obtained from an applied questionnaire, which shows that this approach makes this technology accessible and, in the student's view, brings advantages in their learning.
\end{abstract}

Resumo. O uso de FPGAs tem sido difundido no mercado, por permitir a aceleração de cargas de trabalho que exigem alto poder de processamento. Contudo, o alto custo da compra de ferramentas para a utilização de FPGAs, compromete o ensino dessa tecnologia nas Universidades. Nesse contexto, neste trabalho apresenta-se um estudo sobre a utilização FPGAs em nuvem, no ensino de circuitos integrados reprogramáveis em um curso de Engenharia de Computação. Neste estudo é abordado a elaboração e aplicação de práticas de laboratório. Os resultados obtidos levaram em consideração o feedback obtido de um questionário aplicado, os quais mostram que essa abordagem torna essa tecnologia acessível e, na visão do aluno, traz ganho em sua aprendizagem.

\section{Introdução}

Os estudantes de engenharia devem receber um ensino que os capacite para o mercado de trabalho e para a indústria, que está em constante transformação. A utilização de atividades práticas de laboratório, alinhadas com a realidade de mercado, favorece o crescimento e o preparo do acadêmico para a realidade profissional fora da universidade [Leal et al. 2013]. 
VIII Congresso Brasileiro de Informática na Educação (CBIE 2019)

Anais dos Workshops do VIII Congresso Brasileiro de Informática na Educação (WCBIE 2019)

Porém, esse tipo de atividade demanda material adequado para que se possa alcançar os objetivos didático-pedagógicos planejados. No entanto, muitas vezes para a aquisição desses materiais, tais como placas e licenças de softwares, são necessários recursos financeiros elevados, os quais por vezes não estão ao alcance de universidades públicas.

A disciplina de Sistemas Eletrônicos Digitais Reconfiguráveis (SEDR) do curso de engenharia de computação da Universidade Federal do Ceará (UFC), busca, capacitar o aluno para discutir problemas relacionados à eletrônica digital, bem como fornecer as habilidades necessárias para que este projete sistemas digitais complexos.

Esta disciplina faz uso do ensino de FPGA (Field Programmable Gate Array), que é um tipo de circuito integrado (CI) que pode ser programado para diferentes algoritmos após a sua fabricação, proporcionando conhecimento em desenvolvimento e teste de aplicações para dispositivos lógicos reconfiguráveis. No contexto do referido curso, os FPGAs também são utilizada na disciplina de microprocessadores, em projetos finais de curso.

Com a crescente demanda de processamento de dados gerados pelas inúmeras plataformas e tecnologias do mercado, a utilização de FPGAs se torna cada vez mais vantajosa para acelerar essas cargas de trabalho, por fornecerem alta capacidade computacional e consumo de energia consideravelmente menor do que outros hardwares de propósito especial, como as GPUs [Muslim et al. 2017]. Por esse motivo, torna-se importante trazer esse conhecimento para dentro da academia, estreitando seus laços com o mundo profissional de um engenheiro de computação.

A fim de tornar acessível o uso de uma placa contendo um FPGA high end (FPGAs com tecnologia de ponta), e um ambiente pronto para o desenvolvimento de projetos de FPGAs, a Amazon Web Services (AWS) disponibiliza desde maio de 2017 servidores (também chamados de instâncias) em nuvem que são equipados com FPGAs da tecnologia Virtex da empresa Xilinx e que possuem softwares instalados, como o Vivado e o Sdaccel, que são usados para o desenvolvimento e implementação de soluções para aceleração de hardware. Esse serviço é chamado de Elastic Compute Cloud (EC2) F1. Essa abordagem diminui o custo de um projeto desenvolvido para uma FPGA de alto desempenho como aquela disponível na AWS. O serviço da Amazon custa da ordem de \$USD 1,65 por hora de uso. Além de o custo ser bem razoável quando comparado à aquisição do material, o qual fica obsoleto em poucos anos, a Amazon ainda oferece um voucher de USD\$100,00 para cada aluno de universidades que se cadastrarem no seu sistema.

Outros provedores de nuvem, como Azure e Nimbix Cloud, também disponibilizam FPGAs em suas nuvens. Porém, a Azure não disponibiliza um ambiente de desenvolvimento para aplicações de FPGAs, apenas utiliza o recurso em algumas de suas instâncias, melhorando o poder de processamento [Azure 2019]. Além disso, na época que este estudo foi iniciado o serviço não era disponibilizado na Nimbix Cloud. Por causa disso, o serviço EC2 F1 da AWS foi utilizado como estudo de caso neste trabalho.

O objetivo deste trabalho é realizar um estudo sobre a viabilidade de utilização do serviço EC2 F1, como um recurso didático para a disciplina de Sistemas Eletrônicos Digitais Reconfiguráveis de um curso de graduação em Engenharia da Computação. 
VIII Congresso Brasileiro de Informática na Educação (CBIE 2019)

Anais dos Workshops do VIII Congresso Brasileiro de Informática na Educação (WCBIE 2019)

Por ser um serviço ofertado recentemente, tem-se pouco conhecimento de estudos utilizando as instâncias EC2 F1 como um recurso didático. Essa abordagem está sendo usada pela University of California, Berkeley na disciplina CS 152 Computer Architecture and Engineering, que faz uso das instâncias EC2 F1 em práticas de laboratório [Asanovic 2018].

Em relação ao uso das instâncias EC2 F1, [?] apresenta um estudo que analisa a experiência do uso do AWS EC2 para realizar exercícios de laboratórios em um curso de segurança de computadores e redes. Em [Zhao and Forouraghi 2013], essa tecnologia é utilizada para criar um ambiente virtual de aprendizagem (AVA) para estudantes de Ciência de Computação.

\section{Contextualização e Metodologia}

O trabalho foi realizado no curso de Engenharia de Computação da UFC. As práticas foram aplicadas em dois laboratórios do Departamento de Teleinformática (DETI) durante as aulas da disciplina de Sistemas Eletrônicos Digitais Reconfiguráveis (SEDR), que é um componente optativo da estrutura curricular e é ofertada no sétimo semestre. O total de 8 alunos realizaram as práticas.

A metodologia adotada para a execução deste trabalho é mostrada na Figura 1:

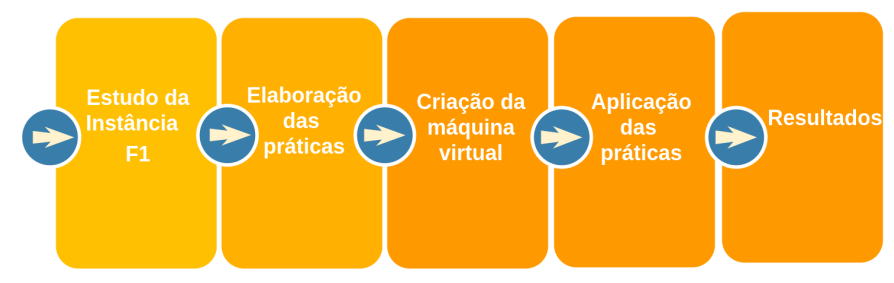

Figura 1. Metodologia

1. Estudo da ementa e plano de ensino da Disciplina de SEDR: Primeiramente, deu-se início ao estudo da ementa e do plano de ensino da disciplina de SEDR para que a utilização das instâncias EC2 F1 fosse adequada da melhor forma possível ao plano de ensino.

2. Estudo da Instância F1: Em seguida, foi realizado um estudo das instâncias EC2 F1 com o intuito de identificar as possibilidades que esse recurso poderia oferecer para o seu uso no ensino de FPGA.

3. Elaboração das práticas: Baseando-se nos estudos já realizados e na documentação da AWS, as práticas de laboratório foram desenvolvidas de forma a oferecer um conhecimento geral do uso das instâncias EC2 F1 como ferramenta, para que o aluno seja capaz de utilizá-la em projetos mais complexos desenvolvidos ao longo da disciplina.

4. Criação da máquina virtual: Com a finalidade de abordar o desenvolvimento local (on-premises) com o kit de desenvolvimento da AWS [Services 2018b] nas práticas 3 e 4, uma máquina virtual foi criada e configurada para conter o kit de desenvolvimento de software (SDK) e Hardware (HDK) da AWS e o ambiente de desenvolvimento da Xilinx que contém a licença da FPGA disponibilizada pela AWS. 
VIII Congresso Brasileiro de Informática na Educação (CBIE 2019)

Anais dos Workshops do VIII Congresso Brasileiro de Informática na Educação (WCBIE 2019)

5. Aplicação das práticas: Após a elaboração das práticas, iniciou-se a fase de aplicação em laboratório.

6. Resultados: Finalmente, nesta última etapa da metodologia foi analisada a efetividade do uso das instâncias EC2 F1, como recurso didático para a disciplina de SEDR através da análise de questionários respondidos pelos alunos ao final de cada prática.

\subsection{Estrutura das aulas de laboratório}

As práticas foram realizadas no Laboratório de Informática, utilizando as máquinas de um laboratório de informática para fazer o acesso remoto às instâncias da AWS. Na Figura 2 é apresentado o procedimento de forma geral de cada atividade de laboratório. Nas práticas 1 e 2 o aluno deve aprender a acessar a instância de desenvolvimento, utilizar o SDK e o HDK e sintetizar um exemplo de projeto na instância com a FPGA. Nas práticas 3 e 4, o aluno deve aprender a utilizar o ambiente de desenvolvimento em uma máquina local, realizando a simulação e síntese do exemplo utilizado nas práticas anteriores. As práticas estão disponíveis em [Rodrigues 2018].

Durante as práticas, os alunos utilizaram o AWS CLi, que é um terminal de comandos disponibilizado pela AWS, para gerar um Design Checkpoint (DCP) e posteriormente uma Amazon FPGA Image (AFI). O primeiro é o resultado da lógica implementada, que é convertida no formato DCP. O segundo utiliza o DCP para gerar um código FPGA compilado que será carregado em uma FPGA disponível em uma instância EC2 F1 [Services 2018a].

Figura 2. Estrutura das práticas.

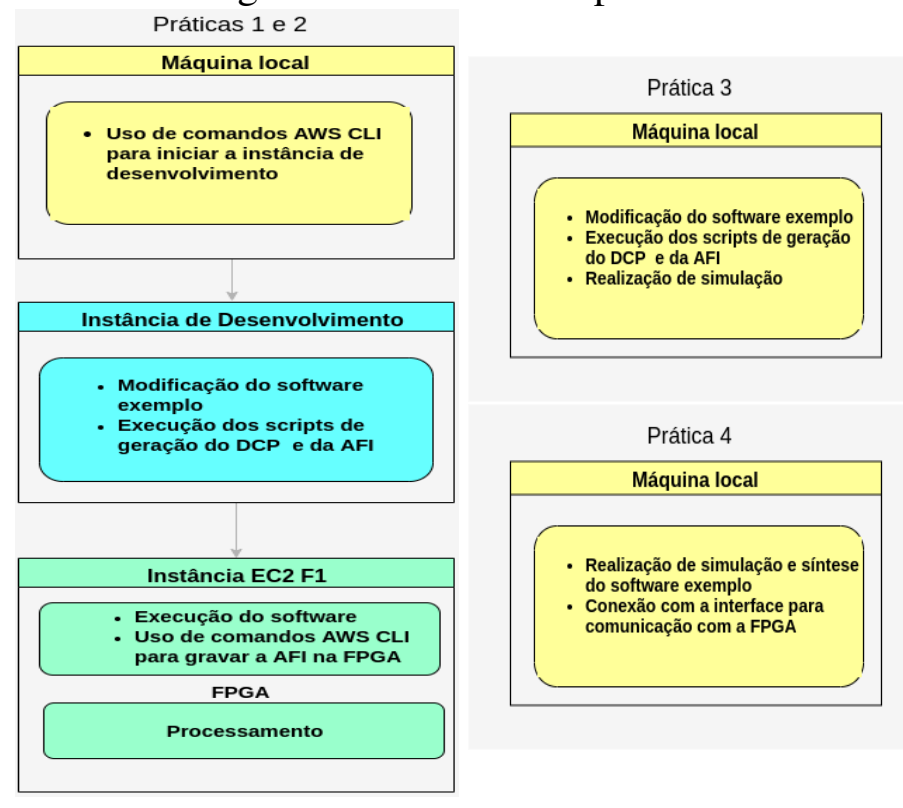

\subsection{Avaliação das práticas de laboratório}

A avaliação da efetividade das práticas, na perspectiva dos alunos, foi feita com base no método utilizado em [Yue et al. 2012].

Para se obter um feedback dos alunos, foram aplicados questionários online, de forma voluntária e anônima, após o término de cada prática, que consistiu de 10 questões. 
VIII Congresso Brasileiro de Informática na Educação (CBIE 2019)

Anais dos Workshops do VIII Congresso Brasileiro de Informática na Educação (WCBIE 2019)

Os alunos responderam a mesma pesquisa ao final de cada aula. Além disso, foi adicionada uma pergunta extra na pesquisa da prática 1, com o objetivo de saber quantos alunos já haviam utilizado algum serviço da Amazon Web Services. O resultado dessa pergunta foi que nenhum aluno da turma havia utilizado a AWS anteriormente.

As questões do questionário foram classificadas em quatro categorias, conforme mostrado no Tabela 1 .

Tabela 1. Questões usadas no questionário.

\begin{tabular}{|c|c|c|}
\hline Categoria & ID & Conteúdo da Pergunta \\
\hline \multirow[t]{2}{*}{$\begin{array}{l}\text { Habilidades com } \\
\text { o Linux e com o } \\
\text { EC2 }\end{array}$} & Q1 & $\begin{array}{l}\text { Por favor, avalie suas habilidades atuais no linux: } \\
\text { Sem Noção Principiante Intermediário Avançado }\end{array}$ \\
\hline & Q2 & $\begin{array}{l}\text { Por favor, avalie suas habilidades atuais no Amazon EC2: } \\
\text { Sem Noção Principiante Intermediário Avançado }\end{array}$ \\
\hline \multirow[t]{2}{*}{$\begin{array}{l}\text { Dificuldade das } \\
\text { Tarefas de } \\
\text { Laboratório }\end{array}$} & Q3 & $\begin{array}{l}\text { As tarefas desses exercícios de laboratório são difíceis. } \\
\text { Discordo Fortemente Discordo Nem concordo nem discordo Concordo }\end{array}$ \\
\hline & Q4 & $\begin{array}{l}\text { Quantas horas você gastou para concluir as tarefas deste laboratório usando a Amazon } \\
\text { EC2? }\end{array}$ \\
\hline \multirow[t]{4}{*}{ O uso do EC2 F1 } & Q5 & $\begin{array}{l}\text { Gostaria de usar o Amazon EC2 F1 em exercícios de laboratório de SEDR semelhantes no futuro. } \\
\text { Discordo Fortemente Discordo Nem concordo nem discordo Concordo Concordo fortemente }\end{array}$ \\
\hline & Q6 & $\begin{array}{l}\text { Essa experiência de uso do Amazon EC2 F1 é útil para meu desenvolvimento de carreira. } \\
\text { Discordo Fortemente Discordo Nem concordo nem discordo Concordo Concordo fortemente }\end{array}$ \\
\hline & Q7 & $\begin{array}{l}\text { Considero importante, para o aprendizado do conteúdo da disciplina, o uso remoto de uma FPGA high end, } \\
\text { considerando que não tenho acesso a FPGA física. } \\
\text { Discordo Fortemente Discordo Nem concordo nem discordo Concordo Concordo fortemente }\end{array}$ \\
\hline & Q8 & $\begin{array}{l}\text { Usaria o Amazon EC2 F1 em pesquisas/trabalhos no futuro. } \\
\text { Discordo Fortemente Discordo Nem concordo nem discordo Concordo Concordo fortemente }\end{array}$ \\
\hline \multirow[t]{2}{*}{$\begin{array}{l}\text { Questões } \\
\text { Abertas }\end{array}$} & Q9 & $\begin{array}{l}\text { Qual é a parte mais difícil em terminar as tarefas neste laboratório usando Amazon } \\
\text { EC2 e por quê? }\end{array}$ \\
\hline & Q10 & $\begin{array}{l}\text { Comentários abertos (por favor, insira quaisquer comentários e sugestões que você desejar } \\
\text { sobre este laboratório e o Amazon EC2 F1). }\end{array}$ \\
\hline
\end{tabular}

\section{Resultados}

Os resultados foram obtidos a partir das respostas do questionário aplicado. A divulgação das respostas dos questionários foi devidamente autorizada pelos alunos.

O questionário foi aplicado quatro vezes, uma vez após cada prática. Os resultados, analisados para cada categoria de perguntas descritas na Tabela 1, são mostrados a seguir.

\subsection{Resultados da pesquisa de Q1 e Q2}

Além do conhecimento relacionado à FPGA, os alunos também precisam possuir ou aprender alguns conhecimentos e habilidades no Linux e Amazon EC2 para realizar as tarefas de cada prática de laboratório. Por isso, as duas perguntas fechadas Q1 e Q2 na primeira categoria pedem aos alunos que avaliem suas habilidades no Linux e no Amazon EC2, respectivamente. Os estudantes deveriam escolher uma das cinco opções de resposta: sem noção, iniciante, intermediário, avançado e guru total.

Os resultados do questionário referentes a essas duas perguntas para cada prática de laboratório são mostrados na Figura 3. Cada coluna mostra a porcentagem de alunos 
VIII Congresso Brasileiro de Informática na Educação (CBIE 2019)

Anais dos Workshops do VIII Congresso Brasileiro de Informática na Educação (WCBIE 2019)

que escolhem cada opção de resposta correspondente a cada prática de laboratório. O nível médio de habilidades em cada prática de laboratório é calculado usando a Equação 1 e anotado sob cada grupo de colunas na Figura 3.

$$
\text { nível medio de habilidades }=\sum_{i=0}^{4}(i * \Delta)
$$

1. Em que $\Delta$ é porcentagem de alunos classificados como nível i.

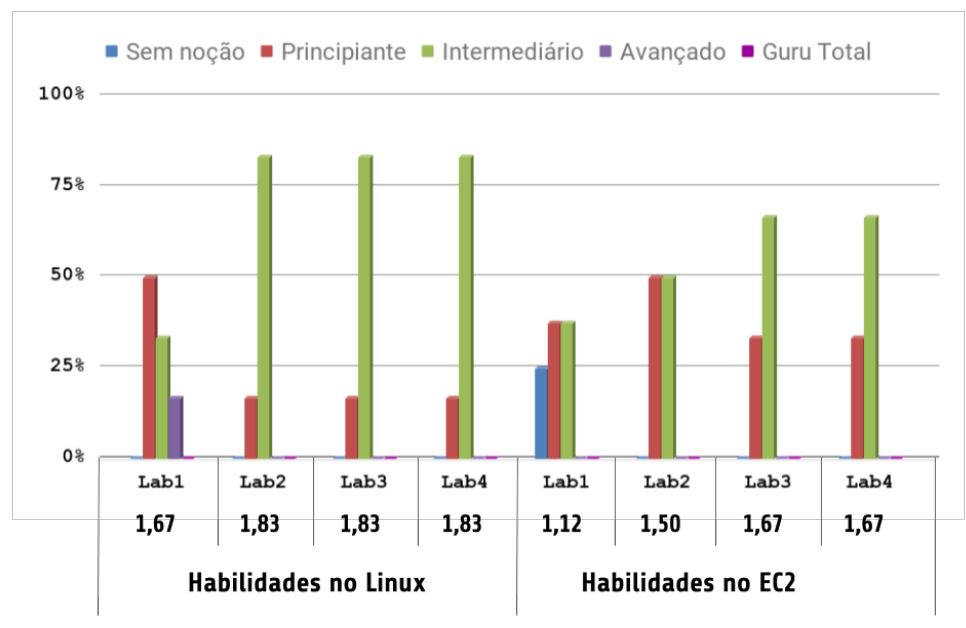

Figura 3. Auto-avaliação do aluno nas habilidades do Linux e do Amazon EC2.

Para obter o nível médio de habilidades, as cinco opções de respostas foram convertidas em valores numéricos, em que o nível 0 significa "Sem noção", o nível 1 significa "Iniciante", o nível 2 significa "Intermediário", o nível 3 significa "Avançado" e o nível 4 significa "Guru total". Não existem escalas de intervalo, já que as respostas são ordinais. Essa conversão foi realizada simplesmente para facilitar a comparação dos níveis de habilidades de uma perspectiva relativa, após cada prática de laboratório.

É possível observar que os exercícios das práticas de laboratório ajudaram o aluno a melhorar suas habilidades de uso do Linux, do EC2 e da AWS em geral. A maioria dos alunos classificaram suas habilidades no Linux como principiante na prática de laboratório 1 . O nível médio de habilidades no Linux aumentou significativamente da prática de laboratório 1 para a prática de laboratório 2 e se manteve constante até a prática 4 . $\mathrm{O}$ nível médio de habilidades no EC2 foi crescente da prática de labotório 1 até a prática de laboratório 3 e manteve-se constante até a prática de laboratório 4. Além disso, apesar de todos os alunos terem declarado que não haviam utilizado algum serviço da AWS anteriormente, após a prática de laboratório 1, uma porcentagem significativa dos estudantes se classificaram como nível intermediário.

\subsection{Resultados da pesquisa de Q3 e Q4}

Na segunda categoria do questionário estão inclusas duas perguntas fechadas, como mostrado na Tabela 1. Para a pergunta três os estudantes deveriam escolher uma das cinco 
VIII Congresso Brasileiro de Informática na Educação (CBIE 2019)

Anais dos Workshops do VIII Congresso Brasileiro de Informática na Educação (WCBIE 2019)

opções de resposta: discordo fortemente, discordo, nem concordo nem discordo, concordo e concordo fortemente. Na Figura 4 é ilustrado o nível médio de dificuldade classificado pelos alunos nas tarefas de cada prática de laboratório e o número médio de horas trabalhadas em cada prática de laboratório. O nível médio de dificuldade é calculado usando a Equação 2.

$$
\text { nível medio de dificuldade }=\sum_{i=0}^{5}(i * \Delta)
$$

2. Em que $\Delta$ é porcentagem de alunos que escolheram o nível i.

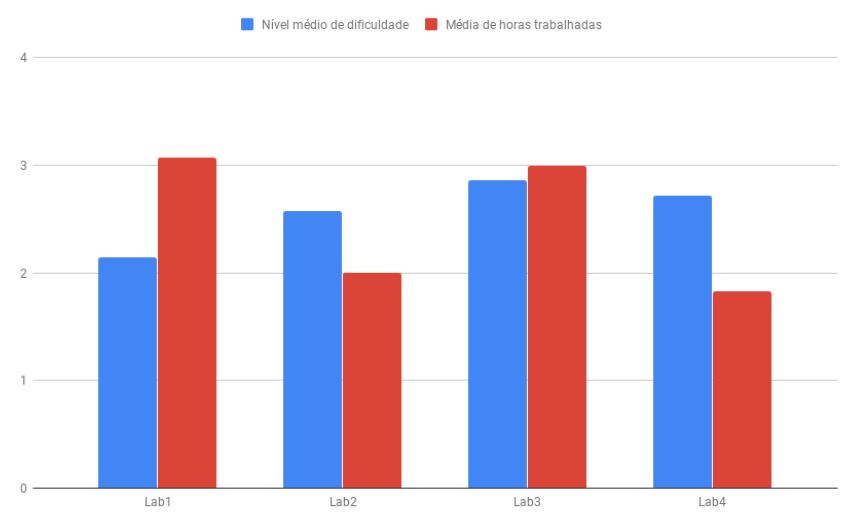

Figura 4. Avaliação da afirmação de que as tarefas em uma prática de laboratório são difíceis

Para obter o nível médio de dificuldade, as cinco opções de respostas foram convertidas em valores numéricos, em que o nível 1 significa "Discordo Fortemente", o nível 2 significa "Discordo", o nível 3 significa "Nem concordo nem discordo", o nível 4 significa "Concordo" e o nível 5 significa "Concordo Fortemente". Podemos ver na Figura 4 que no geral, os valores médio de horas gastas pelos alunos nas práticas de laboratório concorda com os níveis de dificuldade médio avaliado pelos alunos. O nível médio de dificuldade da prática 1 é menor do que o nível médio de dificuldade da prática 2, contudo foram gastas menos horas no término da prática de laboratório 2. Considerando que o nível médio de habilidades do Linux do Amazon EC2 é menor na prática 1, como ilustrado na Figura 3, constata-se que os alunos gastaram uma quantidade extra de tempo no aprendizado de Linux e do Amazon EC2. Os resultados também indicam que a prática de laboratório 3 é a mais desafiadora.

\subsection{Resultados da pesquisa de $\mathrm{Q5}, \mathrm{Q6}, \mathrm{Q7}$ e $\mathrm{Q8}$}

Na terceira categoria, quatro questões Q5, Q6, Q7 e Q8 (conforme a Tabela 1) foram fornecidas ao alunos para obter suas opiniões sobre o uso da instância EC2 F1. Na Figura 5 são ilustrados os resultados obtidos dessas quatro perguntas. Cada coluna representa a porcentagem de estudantes que escolheram a opção de resposta para a pergunta correspondente em cada prática de laboratório, conforme listado na Tabela 1. O nível médio de concordância com o uso do Amazon EC2 é calculado usando a Equação 3 e anotado acima do ID da pergunta, como mostrado na Figura 5. 
VIII Congresso Brasileiro de Informática na Educação (CBIE 2019)

Anais dos Workshops do VIII Congresso Brasileiro de Informática na Educação (WCBIE 2019)

$$
\text { nível medio de concordância }=\frac{1}{4} \sum_{j=1}^{4}\left(\sum_{i=1}^{5}(i * \Delta)\right)
$$

3. Em que $\Delta$ é porcentagem de alunos que escolheram o nível i no lab j.

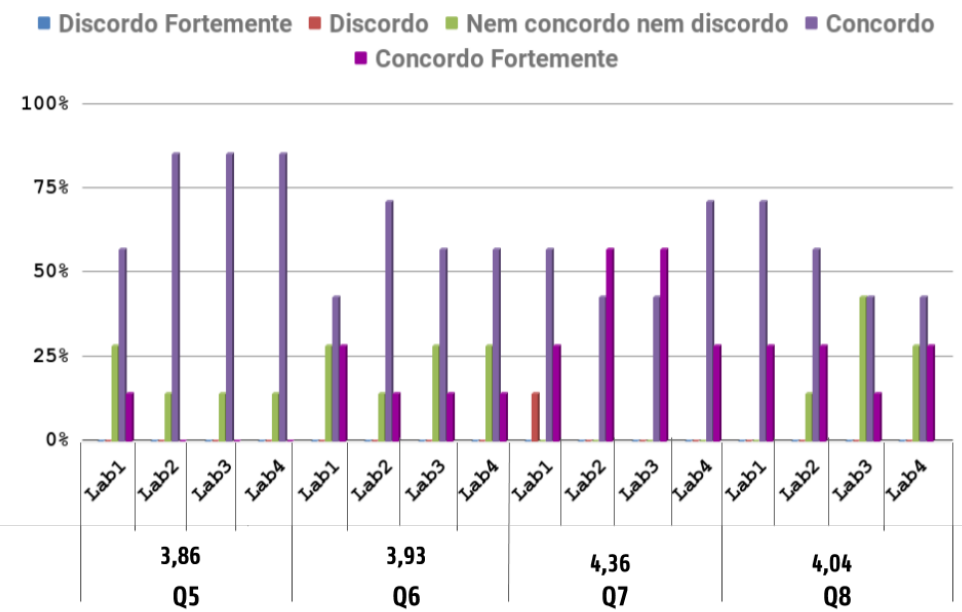

Figura 5. Ranking de concordância com o uso do Amazon EC2.

Para obter o nível médio de concordância, as cinco opções de respostas foram convertidas em valores númericos, de maneira idêntica ao que foi feito na Equação 2. É possível perceber que a porcentagem de alunos que escolheram a opção "Concordo" permance a mais alta em cada prática de laboratório, exceto para a pergunta Q8 na prática de laboratório 3. Nenhum dos alunos escolheu a opção "Discordo fortemente" ou a opção "Discordo", portanto, é possível concluir que a maioria dos alunos gostariam de usar o Amazon EC2 F1 em exercícios de laboratório de SEDR semelhantes no futuro (Q5), considera a experiência de uso do Amazon EC2 F1 útil para o desenvolvimento de suas carreiras (Q6) e usaria o Amazon EC2 F1 em pesquisas/trabalhos no futuro (Q8).

Com relação à afirmação de Q7, na primeira prática de laboratório $14,3 \%$ dos alunos discordam da afirmação, em contrapartida, a porcentagem de estudantes que concordam e concordam fortemente com a afirmação é de $57,1 \%$ e $28,6 \%$, respectivamente. Além disso, a porcentagem dos alunos que concordam fortemente continua a crescer e não há mais alunos que dicordam nas práticas de laboratório seguintes. Com isso, é possível concluir que a maioria dos estudantes concorda com a importância do uso remoto de uma FPGA high end.

Quanto à declaração Q8, 14,3\% dos alunos responderam que nem concordam nem discordam, na prática de laboratório 2. Essa porcentagem aumentou para 42,9\% na prática de laboratório 3 e diminuiu para $28,6 \%$ na prática de laboratório 4 . Com isso, pode-se dizer que alguns alunos podem ter certo receio sobre o uso das instâncias EC2 F1 ou preferem não utilizá-las em outras atividades mesmo depois de terem acumulado experiência nas práticas de laboratório. Portanto, acredita-se que o uso do Amazon EC2 F1 é benéfico dependendo da natureza da pesquisa ou do trabalho que será realizado. 
VIII Congresso Brasileiro de Informática na Educação (CBIE 2019)

Anais dos Workshops do VIII Congresso Brasileiro de Informática na Educação (WCBIE 2019)

\subsection{Resultados da pesquisa de perguntas abertas}

No questionário estão inclusas duas perguntas abertas Q9 e Q10, conforme mostrado na Tabela 1, a fim de permitir que os alunos comentem abertamente sobre o uso do Amazon EC2 F1 nas práticas de laboratório. A pergunta Q9 trata-se da parte mais difícil para concluir as tarefas em cada prática usando a Amazon EC2. Uma opinião comum indicada pelos comentários dos alunos em todas as práticas de laboratório é que a falta de experiência com o uso dos comandos necessários para se utilizar o serviço EC2 F1 torna a prática complicada no primeiro momento, mas o uso contínuo dos comandos torna mais fácil a execução das tarefas das práticas de laboratório. Este resultado é consistente com o progresso do nível médio de habilidades no EC2 ilustrado na Figura 3. Três comentários representativos fornecidos pelos alunos à pergunta Q9 são citados a seguir:

"A principio, por falta de experiência, o passo a passo é um pouco complicado, mas com o uso fica melhor."

"A parte de pedir a instância F1. Mas só porque os comandos são longos e fáceis de confundir. No mais, é tudo muito tranquilo e bem explicado."

"Os primeiros contatos com a interface gráfica e a utilização da máquina virtual, o que não foi problemático."

Houve alguns comentários negativos em resposta à pergunta $\mathrm{Q} 9$, que diz respeito ao tempo disponível para a realização das práticas e a quantidade de tarefas das mesmas. Isso é mostrado nos comentários citados abaixo:

"Muita atividade e pouco tempo."

"Muitas etapas.”

"Tempo curto."

A última pergunta Q10 simplesmente pede que os alunos forneçam quaisquer comentários e sugestões sobre cada prática de laboratório em particular e do Amazon EC2 F1. Houve poucas respostas para essa pergunta, no geral, os alunos ficaram empolgados para exemplos mais complexos na prática de laboratório 1, sugeriram melhoras na descrição da prática e acreditam que com os recursos computacionais adequados, as práticas de laboratório 3 e 4 se tornam ainda mais interessantes. Três comentários representativos fornecidos pelos alunos na pergunta Q10 são citados a seguir:

"Achei muito interessante. Com a eventual melhora dos laboratórios pra fazerem exemplos mais complexos, vai ficar muito bom."

"As práticas em texto, como todas as práticas de texto aplicadas em qualquer disciplina, são um pouco superficiais, não dando um entendimento completo do que se está fazendo. Uma explanação passo a passo antes da realização da prática seria interessante."

"A prática fica legal quando feita com os recursos certos."

\section{Conclusão}

Neste trabalho foi apresentada a experiência do serviço Amazon EC2 F1 como uma ferramenta para o ensino de FPGAs através de quatro práticas de laboratório. 
VIII Congresso Brasileiro de Informática na Educação (CBIE 2019)

Anais dos Workshops do VIII Congresso Brasileiro de Informática na Educação (WCBIE 2019)

Os resultados indicam um aumento do nível médio de habilidades no uso de FPGA em nuvens, utilizando o serviço EC2 F1, e mostram que, de uma maneira geral, os alunos se interessaram em aprender sobre o Amazon EC2 na disciplina, além de considerarem uma experiência útil para o desenvolvimento de suas carreiras.

Observou-se também que a maioria dos alunos não são versados no uso do sistema operacional linux, o que dificulta o aprendizado das instâncias EC2 F1. Embora o objetivo da disciplina de SEDR não seja ensinar o uso do Linux, foi possível observar uma melhoria do conhecimento dos alunos nesse sentido. Com isso, conclui-se que a aplicação das práticas foi efetiva para o aprendizado de FPGA na disciplina de SEDR.

Embora este trabalho tenha como limitação o fato de ter sido realizado com uma amostra pequena $(n=8)$, os resultados qualitativos corroboram com os quantitativos ao perceber-se que essa abordagem, na visão do aluno, traz ganho em sua aprendizagem.

\section{Referências}

Asanovic, K. (2018). CS152 Laboratory Exercise 2 (Version 1.0.2). https : / inst . eecs.berkeley.edu/ cs152/sp18/handouts/lab2-1.0.pdf. [Online; acessado 28-Mar-2018].

Azure (2019). O que são FPGA e como implantar. https://docs . microsoft.com/pt-br/azure/machine-learning/service/ how-to-deploy-fpga-web-service. [Online; acessado 16-Ago-2019].

Leal, A. B., Beloli, A. S. R., Meirinho, C. J., and Weinert, R. L. (2013). Implantação de laboratório de automação da manufatura como meio para melhorar o ensino de automação em curso de engenharia elétrica. In Anais XLI-Congresso Brasileiro de Educação em Engenharia. Gramado: UDESC.

Muslim, F. B., Ma, L., Roozmeh, M., and Lavagno, L. (2017). Efficient fpga implementation of opencl high-performance computing applications via high-level synthesis. IEEE Access, 5:2747-2762.

Rodrigues, V. (2018). Práticas para a disciplina de SEDR do curso de Engenharia de Computação da UFC. https://github.com/vanros/ Praticas-SEDR-AWS /. [Online; acessado 28-Mai-2018].

Services, A. W. (2018a). AWS FPGA - Frequently Asked Questions. https:// github.com/aws/aws-fpga/blob/master/FAQs.md. [Online; acessado 17-Abr-2019].

Services, A. W. (2018b). Enabling on-premises development with Xilinx tools. https://github.com/aws/aws-fpga/blob/master/hdk/docs/ on_premise_licensing_help.md. [Online; acessado 18-Mai-2018].

Yue, C., Zhu, W., Williams, G. L., and Chow, E. (2012). Using amazon ec2 in computer and network security lab exercises: design, results, and analysis. In 2012 ASEE Annual Conference \& Exposition, pages 25-1418.

Zhao, J. and Forouraghi, B. (2013). An interactive and personalized cloud-based virtual learning system to teach computer science. In International Conference on Web-Based Learning, pages 101-110. Springer. 